\title{
Flujos de información y conocimiento en el servicio de referenciación de una biblioteca universitaria
}

\section{Fredy Eduardo Vásquez-Rizo ${ }^{I}$ http://orcid.org/0000-0003-1398-6174}

I Universidad Autónoma de Occidente, Colômbia.

Docente de Planta Facultad de Comunicación y Ciencias Sociales, Universidad Autónoma de Occidente, Colombia. Estudiante de Doctorado en Gestión de la Información y la Comunicación en las Organizaciones, Universidad de Murcia, España. Magister en Ciencias de la Información y Administración del Conocimiento, Instituto Tecnológico y de Estudios Superiores de Monterrey, México.

http://dx.doi.org/10.1590/1981-5344/4078

Este artículo presenta un modelo y una estrategia tendientes a fortalecer el servicio de referenciación en una biblioteca de una universidad colombiana. Para ello, se caracteriza el estado actual de dicho servicio, recurriendo a técnicas cuantitativas y cualitativas, las cuales permiten describir sus componentes y descubrir la necesidad de vincular nuevos elementos para poder satisfacer las necesidades de información de los usuarios. Nuevos componentes que se detectan a partir de un procedimiento de gestión de información y conocimiento aplicado a la comparación entre el estado previo y posterior de un proceso propio del servicio de referenciación y su relación con la asesoría a los usuarios. De esta manera, se concluye que la Sección de Referencia de dicha biblioteca universitaria requiere del modelo y la estrategia propuestos para mejorar su desempeño $y$ 
entregar una respuesta más efectiva a los requerimientos asociados a su labor.

Palavras-chave: Gestión de información y conocimiento. Servicio de referenciación. Flujos de información. Biblioteca universitaria. Información. Conocimiento.

\section{Fluxos de informação e conhecimento no serviço de referência de uma biblioteca universitária}

Este artigo apresenta um modelo e uma estratégia para fortalecer o serviço de referência em uma biblioteca de uma universidade colombiana. Para isso, caracteriza-se o estado atual deste serviço, utilizando técnicas quantitativas e qualitativas, que permitem descrever seus componentes e descobrir a necessidade de vincular novos elementos para atender às necessidades de informação dos usuários. Novos componentes que são detectados a partir de um procedimento de gerenciamento de informações e conhecimento aplicado à comparação entre o estado anterior e posterior de um processo do serviço de referência e sua relação com o aconselhamento do usuário. Desta forma, concluise que a Seção de Referência da referida biblioteca universitária requer que o modelo e a estratégia propostos melhorem seu desempenho e forneçam uma resposta mais eficaz aos requisitos associados ao seu trabalho.

Keywords: Gestão de informação e conhecimento. Serviço de referencia. Fluxos de informação. Biblioteca universitária. Informação. Conhecimento.

Recebido em 16.08.2019 Aceito em 05.10.2020 


\section{Introdução}

Este documento plantea un modelo y una estrategia de gestión de información y conocimiento para efectivizar los procesos realizados en una biblioteca universitaria colombiana, más específicamente en su Sección de Referencia, a partir de sus procedimientos más representativos relacionados con la satisfacción de las necesidades de información de sus usuarios.

Para ello, se realiza la modelación del sistema de referenciación actual, la caracterización de los procesos implicados, la identificación de los tipos de información y de conocimiento existentes y la presentación de una propuesta para mejorar los procedimientos mencionados, con la intención de fortalecer dicha Sección $y$, por ende, a la biblioteca y a la institución.

De esta forma, se pretende que la Sección de Referencia pueda mejorar de manera significativa sus procesos, gracias a la conformación de un modelo estratégico que sustente su oferta de servicios y que garantice el cumplimiento efectivo de su labor, brindando un mejor servicio a sus usuarios, a partir de un proceso oportuno de difusión y transformación de información y conocimiento.

Es así como el presente artículo pretende explicitar el conocimiento tácito presente en el Capital Humano adscrito a la Sección de Referencia de la institución objeto de estudio, con el objetivo de proponer una estrategia para dicho fin, que implique el diseño de un modelo, la definición de funciones y perfiles laborales asociados a la dependencia (usando como ejemplo un procedimiento específico) y la mejora en el servicio y en el flujo de información.

\section{Revisión de la literatura}

\subsection{Las bibliotecas universitarias y la función de referenciación}

Las bibliotecas universitarias son departamentos de servicio, que han ido ganando espacio paulatinamente en las Instituciones de Educación Superior - IES, pues han pasado de ser simples depósitos de libros y salas de estudio a dependencias neurálgicas (GAVILÁN, 2008), encargadas de producir conocimiento a partir de información, ayudando a conformar el acervo cognitivo de la Universidad de hoy, siendo el escenario perfecto para la utilización de las Tecnologías de la Información y la Comunicación - TIC asociadas a la recuperación de información (ITO; HATANO; KUMAGAI, 2018; BHATT, 2001).

Sin embargo, en muchas instituciones aún no se le da a esta dependencia el reconocimiento que se merece (GONZÁLEZ-FERNÁNDEZ- 
VILLAVICENCIO; CÁNOVAS-ÁLVAREZ; ARAHAL-JUNCO, 2014), puesto que no se considera que la biblioteca y sus secciones deban contar con un Capital Humano preparado idóneamente, pues se presume que sus funciones pueden ser realizadas por cualquier persona que sepa medianamente organizar libros y buscar información.

Es por esto que es muy común encontrar en las universidades, especialmente en la Sección de Referencia, a personas con competencias distantes de la función de referenciación, en ocasiones suplida por estudiantes, auxiliares, monitores o profesionales no especializados en el área, quienes reciben una capacitación fugaz, sin profundidad, lo que repercute finalmente en detrimento del servicio (EVERALL; LOGAN, 2017).

Situación que se hace evidente cuando el usuario no logra ubicar la información que necesita y no encuentra un apoyo especializado en el "referencista", lo que ocasiona que quede insatisfecho con el servicio de la biblioteca $y$, por ende, de la institución, teniendo que recurrir a verdaderos bibliotecólogos profesionales en el campo, tales como el Coordinador de Procesos Técnicos o el Director, quienes no tienen el tiempo suficiente para la atención. De ahí la importancia de contar con personas competentes en esta Sección (MACDONALD, 2018).

Por lo anterior, las IES y sus bibliotecas se han visto en la obligación de diseñar, desarrollar e implementar mecanismos para resolver este inconveniente y han recurrido para ello a la construcción de estrategias y modelos especializados, empleando para esto toda la práctica y teoría asociadas a la gestión de la información y del conocimiento, con el fin de contar con un insumo que les permita fortalecer sus procesos y seleccionar, ubicar y capacitar adecuadamente a su personal (WEXLER, 2002).

\subsection{La gestión de información y de conocimiento en las bibliotecas universitarias}

Dentro de la estructura de las bibliotecas universitarias se manejan dos tipos de información y conocimiento principalmente: tácitos y explícitos. Los primeros, presentes en las mentes de los actores que intervienen en sus diversos procesos, pero ausentes de un constructo común, por no haber sido aún compartidos. Y los segundos, presentes en algunos documentos y en el saber hacer habitual de estos mismos actores, pero carentes de sentido cuando la dependencia no cuenta con las estrategias apropiadas para su transferencia, procesamiento, difusión y co-creación (YEN, 2009).

Es por esto que la gestión de información y conocimiento aparece como aquel procedimiento que puede hacer posible la explicitación de aquellos elementos tácitos y la articulación de los explícitos, con miras a la 
potenciación del espacio que los contiene ( $y$ de las personas que los utilizan) y a la conformación de sinergias que permitan la realización más eficiente de los diferentes procesos (VÁSQUEZ-RIZO; RODRÍGUEZMUÑOZ; GÓMEZ-HERNÁNDEZ, 2019; VIDAL-LEDO; ARAÑA-PÉREZ, 2012).

En el caso del servicio de referenciación realizado en dichas bibliotecas, estos procesos se refieren a diligenciar las planillas de servicio, registrar el material bibliográfico, asesorar a los usuarios, recoger el material de circulación y préstamo, organizar documentos, entre otros (GONZÁLEZ-FERNÁNDEZ-VILLAVICENCIO, 2012; WALKER; JANES, 1999), los cuales implican una ardua labor, que requiere de personas conocedoras de cada proceso y de su implicación en el andamiaje asertivo del servicio.

De esta manera, la gestión de información y conocimiento aparece para soportar aquellas estrategias que permitan llevar a buen término la función de referenciación, donde cada persona implicada en cada uno de estos procesos, desde el jefe de sección, pasando por el empleado referencista, hasta el usuario, pueda hacer uso de la información y el conocimiento colectivos, al mismo tiempo que participa en su construcción (KHURSHID; ZHENG; MUHAMMAD, 2019). Todo ello en procura de un mejor servicio, soportado en el aprovechamiento de los insumos asociados a esta rama de la gestión.

\section{Metodología}

El desarrollo de este estudio se hace a partir de un enfoque mixto (cuantitativo y cualitativo) (CIENFUEGOS-VELASCO; CIENFUEGOSVELASCO, 2016; DE PELEKAIS, 2000), asociado a la teoría de la gestión de la información y el conocimiento. Los datos utilizados para el análisis cuantitativo se derivan de los resultados numéricos obtenidos mediante la aplicación de encuestas, que involucran variables convenidas previamente con la IES.

Para la aplicación de las encuestas, se han seleccionado distintos grupos de actores (usuarios, empleados, jefes) y diferentes formatos, pues cada uno de ellos tiene una relación distinta con la Sección de Referencia, y su complementariedad puede enriquecer el estudio, posibilitando una mirada holística de todo el proceso (HUVILA; AHMAD, 2018).

En todas las encuestas desarrolladas se pidió que los encuestados calificaran cada pregunta de 1 a 5 , siendo 5 el máximo valor posible (decisión que facilita la medición cuantitativa, la aplicación de procesos estadísticos, el establecimiento de tendencias y el camino a seguir en los grupos focales). A continuación, se presentan algunas preguntas representativas, de acuerdo con cada público objetivo: 
a) Usuarios: ¿Se hallan en la Sección de Referencia los documentos que necesita?, ¿Encuentra atención oportuna y adecuada a sus necesidades de información?, ¿Son contestadas adecuada y amablemente, por parte de los referencistas, las preguntas que formula?, ¿Conoce las políticas, directrices y normativas que regulan el servicio de referencia?, ¿Sabe cuál es el horario de atención de la Sección de Referencia?, ¿Considera que la capacidad de los referencistas es adecuada para solucionar sus necesidades de información?, ¿Ha tenido que recurrir a otras secciones para resolver sus dudas de información?

b) Empleados: ¿Conoce los objetivos generales de la Sección?, ¿Considera la Sección a los usuarios a la hora de fijar sus objetivos?, ¿Cree que los usuarios se sienten complacidos con el servicio prestado?, ¿Considera que está capacitado para las funciones de su labor?, ¿Se le pregunta sobre sus necesidades de formación?, ¿Cree que la Sección está preparada para adaptarse a los cambios de la profesión?, ¿Considera que su jefe se siente satisfecho con su labor?

c) Jefes: ¿Son idóneas para el cargo las personas encargadas del servicio de referencia?, ¿Cuenta con algún sistema de indicadores o de evaluación para el desempeño de los referencistas?, ¿La Sección de Referencia posee los recursos necesarios para la prestación adecuada del servicio?, ¿Es suficiente el personal de referencia para atender las necesidades regulares de información de los usuarios?, ¿Cree que se debe capacitar permanentemente a los empleados de la Sección de Referencia?, ¿Considera que las directivas de la biblioteca y la universidad son conscientes de la importancia de contar con una Sección de Referencia mejor estructurada?, ¿Se necesita una nueva estrategia para formalizar y efectivizar la labor en la Sección de Referencia?

En términos cualitativos, se realizan entrevistas grupales con los actores más representativos de los distintos grupos de análisis, con la intención de complementar, justificar o refutar algunas tendencias observadas en las respuestas a las encuestas (PATTON, 2014). Todo esto posibilita explicitar los conocimientos tácitos de los sujetos de estudio, en relación con el proceso actual y el deseado, asociado a la Sección de Referencia.

Todo lo anterior permite detectar que evidentemente existe un problema asociado a las funciones del personal de la Sección de Referencia, debido a que sus labores no se realizan de forma especializada 
o adecuada, razón relacionada con la falta de idoneidad de las personas a cargo y al desconocimiento del campo. Situaciones que fortalecen la hipótesis asociada a la necesidad de una estrategia y un modelo, construidos a partir de las necesidades detectadas a través de la aplicación de las técnicas mencionadas, que conviertan la información y el conocimiento tácito de las personas implicadas en el proceso de referenciación en un conocimiento explícito y compartido, incorporándolo en la memoria institucional (MUSKAT; DEERY, 2017).

\section{Resultados}

\subsection{Modelación de la estructura actual}

El anterior procedimiento permitió identificar y conocer el Capital Humano implicado en la Sección de Referencia de la biblioteca universitaria objeto de estudio (y su percepción). Esto posibilitó entender cuál es la conformación actual de la estructura que rige dicha dependencia (adicionalmente su Capital Estructural y su Capital Relacional). De esta manera, se pudo elaborar un modelo que expresó dicho conjunto (Figura 1 ), el cual evidenció algunos aspectos positivos, pero también algunos aspectos a mejorar o adicionar.

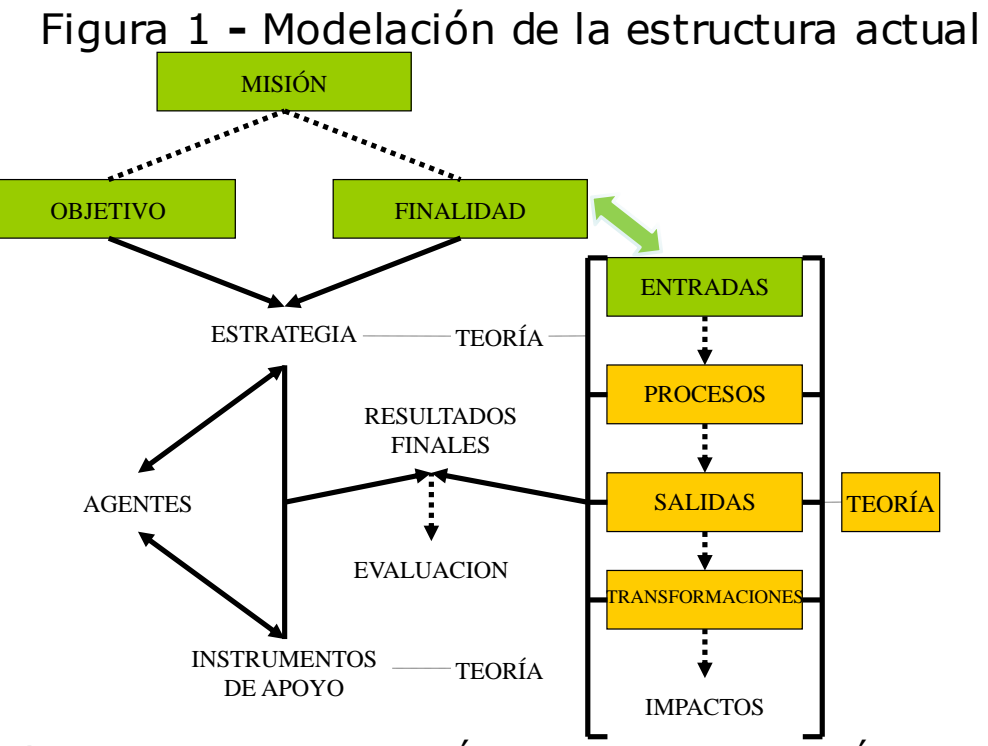

Fuente: elaboración propia, con base en VÁSQUEZ-RIZO; GABALÁN-COELLO, 2011)

Cada componente existente actualmente se enmarcó dentro de un cuadro con un color diferenciador, de acuerdo con su grado de existencia en la Sección de Referencia (verde, existe y es apropiado; amarillo, existe, pero necesita ser fortalecido o transformado, en la mayoría de los casos), mientras que por fuera de los cuadros, y a manera de información, quedaron otros posibles componentes, los cuales serán detallados más adelante, en la solución propuesta. Nótese que la flecha verde simboliza lo 
abrupto o distante de la relación actual entre los procesos y los componentes fundamentales.

A continuación, se describe cada uno de estos componentes, a partir de los agentes, flujo, actividades y requerimientos detectados, y se presentan algunas especificaciones dadas de acuerdo a sus procedimientos (representados, posteriormente, en diagramas de flujo de información y conocimiento).

a) Componente Misión: enfatiza en la posibilidad de crear condiciones misionales en la Sección, en relación con la consolidación, flujo y socialización de la información y el conocimiento contenidos en sus actividades, sirviendo como insumo para la estrategia y el modelo, a partir de la validación de los resultados y su implementación y difusión.

b) Componente Objetivo: hace mención al objeto del estudio. Es decir, explicitar la información y el conocimiento tácitos de los miembros de la Sección de Referencia y articularlos, con la intención de proponer una estrategia, que implique el diseño de un modelo y la mejora en el servicio.

c) Componente Finalidad: representa mejorar el servicio de la Sección de Referencia. Es decir, lo que se pretende lograr mediante la implementación de la estrategia y la elaboración del modelo.

d) Componente Entradas: hace alusión al conocimiento que posee la Sección de Referencia en cuanto al reconocimiento de su actual personal (sin profundizar aún en sus características) y demás recursos.

e) Componente Procesos: hace referencia a todas aquellas actividades que se realizan en la Sección (registrar y ubicar material bibliográfico, asesorar usuarios, etc.), y que necesitan mantenerse u optimizarse para obtener los mejores resultados.

f) Componente Salidas: representa todos aquellos productos que pueden surgir del proceso desarrollado.

g) Componente Transformaciones: implica todos aquellos cambios en los conocimientos, actitudes, habilidades, capacidades tecnológicas, etc., de los empleados de la Sección, gracias a la implementación del proceso descrito y sus resultados.

h) Componente Teoría: reúne los principales fundamentos teóricos asociados a la realización de lo propuesto.

Una vez descritos los componentes estructurales actuales de la Sección de Referencia, a manera de ejemplo, se procede a presentar un 
diagrama de flujo de uno de sus procesos más representativos: "asesorar usuarios en referenciación" (Figura 2). Aquí, cada figura geométrica simboliza un agente, elemento o característica dentro del proceso (el óvalo, al Referencista; el triángulo, al Jefe; la flecha, al Proceso concreto, y el rombo, al Usuario).

Y su explicación es que al carecer de un esquema procedimental efectivo (producto de la estructura actual), el proceso presenta algunos vacíos, debido a que no existe claridad en su realización de forma eficiente, situación que interrumpe la relación Referencista-Usuario. En este proceso el Jefe debería funcionar como elemento externo, pero debe intervenir para poder solucionar los vacíos producidos por el rompimiento de dicha relación.

Figura 2 - Diagrama de flujo actual para el proceso "asesorar usuarios en referenciación"

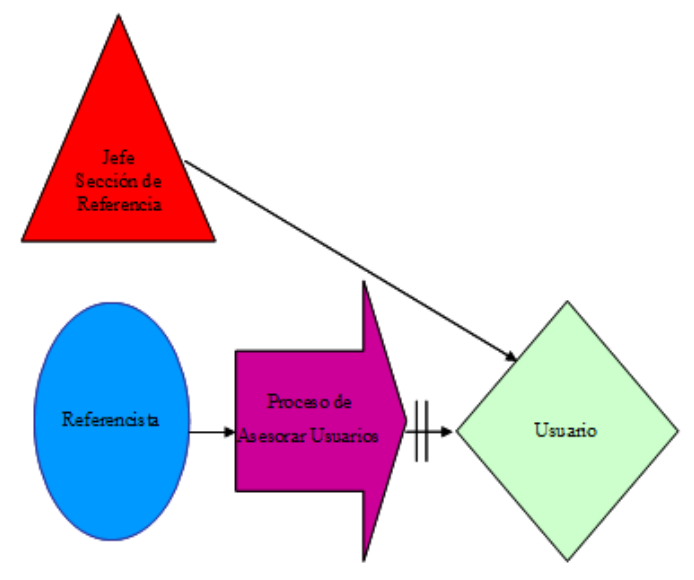

Fuente: elaboración propia

En relación con la estructura actual, si bien el Componente Objetivo es claro, pues se intenta dar una asesoría útil al usuario y éste se respalda con la existencia, por lo menos en lo procedimental u operativo, de los componentes Entradas, Procesos y Salidas, y posiblemente con el Componente Teoría, el esquema falla en el Componente Transformaciones, producto de no realizar correctamente el proceso y seguirlo haciendo de esa forma, a pesar de saberlo y reconocerlo (esto se evidenció en las encuestas y entrevistas), lo que repercute en el mediano o nulo cumplimiento de los componentes Misión y Finalidad, en detrimento de un buen servicio.

Como se puede apreciar, en esta Sección se presentan numerosas relaciones asociadas al flujo de la información y el conocimiento (Referencista-Usuario, Referencista-Proceso, Referencista-Jefe, ProcesoUsuario, Jefe-Usuario), las cuales no se desarrollan de la mejor manera, precisamente por las fallas en el proceso y en la estructura actual de la 
Sección. Situación que podría corregirse a partir de la explicitación de dicha información y conocimiento (LUSTIG; HAIDER, 2019; GASPAR et al., 2009), a partir de la estructura y el modelo propuestos.

\subsection{La solución propuesta}

Con base en lo anterior, a continuación, se exponen algunos aspectos tendientes a fortalecer la estructura actual de la Sección de Referencia de la biblioteca universitaria objeto de análisis. Información que debe estar incorporada en el modelo, con la intención de formalizar la actividad de referenciación (GUITARD, 2013).

Dichos aspectos se presentan en forma de componentes en la Figura 3 , representados en color rojo. Nótese que ya no aparece la flecha en color verde, pues con los componentes adicionales ésta se reemplaza y la relación entre el proceso y los componentes fundamentales se fortalece.

Figura 3 - Modelación de la estructura propuesta

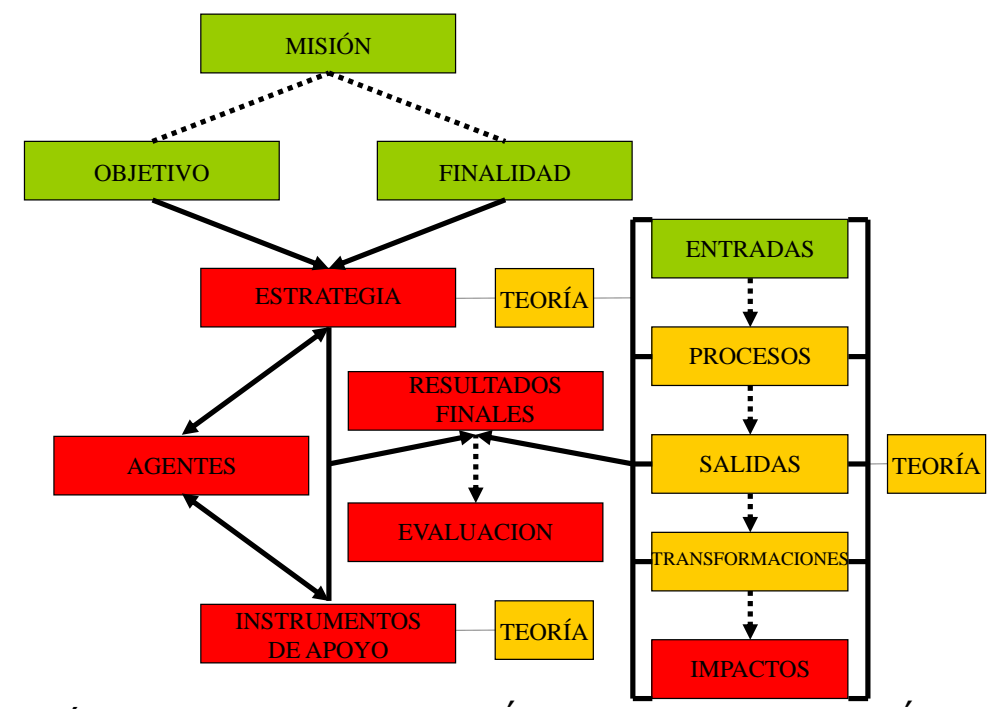

Fuente: elaboración propia, con base en VÁSQUEZ-RIZO; GABALÁN-COELLO, 2011)

Y ahora se describen los nuevos componentes:

i) Componente Impactos: establece la utilidad del proceso, así como su viabilidad de replicación, determinando los impactos, tanto internos como externos, del trabajo realizado.

j) Componente Estrategia: considera la forma cómo se va a realizar la implementación del servicio, con la intención de solventar cualquier problema, pensando en la obtención de los mejores resultados. 
k) Componente Agentes: con base en el Componente Entradas, posibilita conocer la caracterización del Capital Humano participante en el servicio, así como sus formas de interacción.

I) Componente Instrumentos de Apoyo: reúne todo el Capital Estructural (instrumentos, herramientas, indicadores, etc.) que requiere el servicio.

m) Componente Resultados Finales: si bien es claro que el resultado esperado es la mejora del servicio de referencia, también pueden surgir algunos otros (replicación de la solución propuesta en otras secciones o dependencias, informes y reportes, batería de indicadores, productos, etc.).

n) Componente Evaluación: considera la revisión de las interrelaciones entre cada componente del modelo (Capital Relacional), permitiendo medir y valorar su participación, implementación y efectividad, velando por el correcto desempeño de la función de referenciación y de la solución propuesta.

Ahora, se expone el respectivo diagrama de flujo para el proceso "asesorar usuarios en referenciación" (Figura 4), según los nuevos componentes del modelo:

Figura 4 - Diagrama de flujo propuesto para el proceso "asesorar usuarios en referenciación"

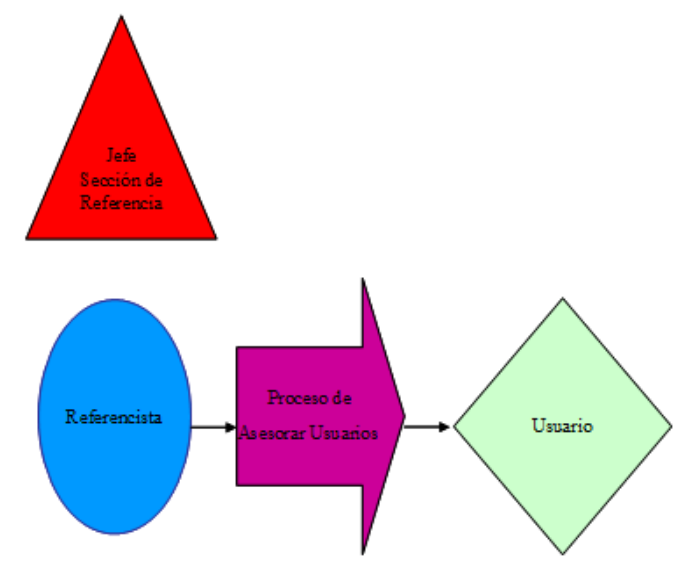

Fuente: elaboración propia

En relación con la estructura propuesta, se intentan corregir los componentes defectuosos en el anterior esquema (Figura 2) (Transformaciones, Misión y Finalidad), preservando aquellos que se "desarrollaban correctamente" (Objetivo, Entradas, Procesos, Salidas y Teoría -este último apareciendo en cualquier instancia-), a partir de la incorporación de los nuevos componentes. 
De esta forma, se plantea una nueva manera de realizar el proceso, desde la capacitación del Referencista (o su contratación), como parte activa de una nueva estrategia (Componente Estrategia), que implica un Capital Humano más pertinente (Componente Agentes), soportado en un Capital Estructural propicio (Componente Instrumentos de Apoyo), en procura de unos resultados (Componente Resultados Finales) tendientes a ser revisados permanentemente (Componente Evaluación), generando unos impactos apropiados asociados al proceso (Componente Impactos).

Como se puede observar, con la estrategia y el modelo propuestos, el proceso "asesorar usuarios en referenciación" adquiriría un "estado ideal", pues se definirían los protocolos y procedimientos de trabajo efectivo y eficiente, mejorando la relación Referencista-Usuario. Y de esta manera, el Jefe regresaría a su "estado natural", siendo un elemento externo al proceso, evitando asumir funciones que no le corresponden.

\section{Discusión}

A través de la estrategia y el modelo propuestos, la Sección de Referencia de la biblioteca universitaria analizada podría llegar a fortalecerse en los siguientes aspectos: brindar un mejor servicio a los usuarios, más eficiente y confiable; aprender a trabajar colaborativamente; comprender la importancia de compartir la información, transformarla y transferir el conocimiento; romper paradigmas, especialmente aquel que apunta a atesora información y conocimiento; reconocer la presencia de conocimientos tácitos y explícitos en la Sección, identificándolos y empleándolos para su perfeccionamiento; aprender a distribuir funciones y responsabilidades de manera efectiva; propender por el bien común y el sentido de pertenencia.

Adicional a esto, participar activamente en la cultura institucional; ser pionera en procesos de gestión de información y conocimiento; reconocer a la información y al conocimiento como elementos generadores de ventaja competitiva y factores de desarrollo; fortalecer las relaciones suscitadas al interior de sus procesos; aprovechar al máximo sus capitales conformantes (Humano, Estructural y Relacional), y contar con las personas idóneas para sus procesos.

\section{Conclusiones}

Después de haber hecho el análisis del servicio de referencia en una biblioteca universitaria, usando estrategias de investigación cuantitativa y cualitativa, se puede afirmar que el modelo propuesto permite prestar un mejor servicio de referenciación, acorde con las necesidades actuales de información de los usuarios y con los intereses de la Sección, la biblioteca y la institución. 
Se espera que las directivas de la biblioteca y la institución, una vez reconozcan las posibilidades de desarrollo expuestas, socialicen la estrategia, el modelo y cada uno de sus mecanismos y componentes, para que éstos se instauren en la memoria organizacional de la biblioteca y la Sección, así como en la cultura de los usuarios y referencistas. Para esto último, es evidente que se requiere que el cargo de referencista sea desempeñado por personas idóneas y en permanente proceso formativo.

Se recomienda que la biblioteca realice constantemente un seguimiento al desarrollo aquí expuesto, una vez éste sea implementado en sus instalaciones, con el fin de establecer correctivos y tomar decisiones estratégicas en el momento preciso y evitar así imprecisiones o especulaciones que no permitan llegar a los resultados propuestos.

Es muy importante profesionalizar en la universidad el proceso de referenciación, así como ordenar y formalizar cada una de las funciones que estos profesionales realizan, pues esta Sección es una de las áreas que mayor contacto tiene con el usuario de la biblioteca, por lo que debe ser prioridad responder eficaz y efectivamente a las necesidades de información y referenciación que éste requiere.

Como se puede observar, pueden ser muchos los impactos o beneficios que pueden entregar esta estrategia y este modelo de gestión de información y conocimiento; sin embargo, será responsabilidad de las personas encargadas de tomar decisiones institucionales la operatividad e implementación de lo aquí expuesto, y de utilizar a la información y al conocimiento como motores propulsores del desarrollo institucional.

\section{Referências}

BHATT, G. D. (2001). Knowledge management in organizations:

examining the interaction between technologies, techniques and people. Journal of Knowledge Management, Bingley, v. 5, n. 1, p. 68-75, mar. 2001. https://doi.org/10.1108/13673270110384419

CIENFUEGOS-VELASCO, M. A. ; CIENFUEGOS-VELASCO, A. Lo cuantitativo y cualitativo en la investigación. Un apoyo a su enseñanza. Revista Iberoamericana para la Investigación y el Desarrollo Educativo, Guadalajara, v. 7, n. 13, p. 1-22, dic. 2016. Disponible en: http://www.scielo.org.mx/scielo.php?script=sci_arttext\&pid=S200774672016000200015 . Acceso en el: 14 agosto 2019.

DE PELEKAIS, C. Métodos cuantitativos y cualitativos: diferencias y tendencias. Telos: Revista de Estudios Interdisciplinarios en Ciencias Sociales, Madrid, v. 2, n. 2, p. 347-352, ene. 2000. Disponible en: http://ojs.urbe.edu/index.php/telos/article/view/997/943. Acceso en el: 4 agosto 2019. 
EVERALL, K.; LOGAN, J. A mixed methods approach to iterative service design of an in-person reference service point. Evidence Based Library \& Information Practice, Montréal, v. 12, n. 4, p. 178-185, dic. 2017. https://doi.org/10.18438/B87Q2X

GASPAR, M. A.; DONAIRE, D.; DOS SANTOS, S. A.; SILVA, M. C. Um etudo dos portais corporativos como instrumento de externalização do conhecimento explícito em universidades. Revista Brasileira de Gestão de Negócios, São Paulo, v. 11, n. 31, p. 119-133, jun. 2009. Disponible en: https://dialnet.unirioja.es/servlet/articulo?codigo=3146831. Acceso en el: 6 agosto 2019.

GAVILÁN, C. M. Bibliotecas universitarias: concepto y función. Los CRAI. Disponible en: http://eprints.rclis.org/14816/1/crai.pdf. Acceso en el: 6 agosto 2019.

GONZÁLEZ-FERNÁNDEZ-VILLAVICENCIO, N. Servicios de referencia en bibliotecas universitarias: tendencias y plan de marketing. El Profesional de la Información, Barcelona, v. 21, n. 6, p. 567-576, dic. 2012. https://doi.org/10.3145/epi.2012.nov.03.

GONZÁLEZ-FERNÁNDEZ-VILLAVICENCIO, N. ; CÁNOVAS-ÁLVAREZ, E.; ARAHAL-JUNCO, C. Evaluación del servicio de referencia de una biblioteca universitaria: Biblioteca de la Universidad de Sevilla. Revista Española de Documentación Científica, Madrid, v. 37, n. 2, p. 1-17, oct. 2014. https://doi.org/10.3989/redc.2014.2.1072.

GUITARD, L. A. Indexation par sujet en archivistique et en bibliothéconomie: du pareil au même? Documentation \& Bibliotheques, Montréal, v. 59, n. 4, p. 201-212, dic. 2013. https://doi.org/10.7202/1019217ar.

HUVILA, I.; AMAD, F. Holistic information behavior and the perceived success of work in organizations. Library and Information Science Research, Norman, OK, v. 40, n. 1, p. 18-29, ene. 2018. https://doi.org/10.1016/j.lisr.2018.03.004.

ITO, A.; HATANO, H.; KUMAGAI, M. How to convert skills of masters to explicit knowledge through the use of ICT: In the case of reparing dent in a car. Proceedings of the 9th IEEE International Conference on Cognitive Infocommunications (CogInfoCom) Cognitive Infocommunications (CogInfoCom), Budapest: Institute of Electrical and Electronics Engineers - IEEE, 2018.

https://doi.org/10.1109/coginfocom.2018.8639928

KHURSHID, A.; ZHENG, J.; MUHAMMAD, R. Assessing the literature of knowledge management (KM) in the field 
of library and information science. Information Discovery and Delivery, London, v. 47, n. 1, p. 35-41, mar. 2019. https://doi.org/10.1108/IDD06-2018-0021

LUSTIG, C.; HAIDER, H. Response-effects trigger the development of explicit knowledge. Acta Psychologica, Amsterdam, v. 194, p. 87-100, mar. 2019. https://doi.org/10.1016/j.actpsy.2019.01.016

MACDONALD, $\mathrm{H}$. Undergraduate students can provide satisfactory chat reference service in an academic library. Evidence Based Library \& Information Practice, Edmonton, v. 13, n. 2, p. 112-114, jun. 2018. https://doi.org/10.18438/eblip29414

MUSKAT, B.; DEERY, M. Knowledge transfer and organizational memory: An events perspective. Event Management, Putnam Valley, NY, v. 21, n. 4, p. 431-447, nov. 2017.

https://doi.org/10.3727/152599517X14998876105765

PATTON, M. Q. Qualitative research \& evaluation methods. Integrating theory and practice (4th. ed.). Thousand Oaks, CA: Sage Publications, 2014

VÁSQUEZ-RIZO, F. E.; GABALÁN-COELLO, J. Implementación de un modelo de administración de capital humano en un grupo de investigación. El Profesional de la Información, Barcelona, v. 20, n. 5, p. 516-526, oct. 2011. https://doi.org/10.3145/epi.2011.sep.05

VÁSQUEZ-RIZO, F. E.; RODRÍGUEZ-MUÑOZ, J. V.; GÓMEZ-HERNÁNDEZ, J. A. La gestión de información para medir la capacidad investigadora en una institución de educación superior. Revista Espacios, Caracas, v. 40, n. 8, p. 18-30, mar. 2019. Disponible en:

<https://revistaespacios.com/a19v40n08/19400818.html >. Acceso en el: 30 julio 2019.

VIDAL-LEDO, M. J.; ARAÑA-PÉREZ, A. B. Gestión de la información y el conocimiento. Educación Médica Superior, La Habana, v. 26, n. 3, p. 474484, sep. 2012. Disponible en:

http://scielo.sld.cu/scielo.php?script=sci_arttext\&pid=S0864-

21412012000300013. Acceso en el: 30 julio 2019.

WALKER, G.; JANES, J. Online retrieval: a dialogue of theory and practice (2nd Ed.). Englewood, CO: Libraries Unlimited, 1999.

WEXLER, M. N. Organizational memory and intellectual capital. Journal of Intellectual Capital, London, v. 3, n. 4, p. 393-414, dic. 2002. https://doi.org/10.1108/14691930210448314 
YEN, B. Coordinated combination of knowledge in a KM system to improve work effectiveness and efficiency: A knowledge sharing approach for library reference service. Proceedings of the 6th International Conference on Intellectual Capital, Knowledge Management \& Organizational Learning, Montréal: McGill University, 2009. 